\title{
Robust spatiotemporally integrated fractionation in radiotherapy
}

\author{
Ali Ajdari, Archis Ghate*
}

May 24, 2016

\begin{abstract}
Spatiotemporally integrated fractionation involves finding a fluence-map and a number of treatment sessions that maximize tumor-kill subject to dose-limits on organs-at-risk (OAR). This problem was recently formulated using the linear-quadratic dose-response model. Owing to the uncertainty in dose-response parameters, however, a solution presumed optimal might be infeasible in practice. We address this via a robust counterpart and its convex reformulation wherein the price of robustness is small and robust solutions are less infeasible than nominal even outside our uncertainty sets.
\end{abstract}

Keywords: Intensity Modulated Radiation Therapy; convex optimization; linear programming duality.

\section{Introduction}

The goal in external beam radiotherapy is to maximize damage to the tumor while limiting toxic effects of radiation on nearby organs-at-risk (OAR). This is achieved via spatial localization and temporal dispersion (called fractionation) of radiation dose.

On the spatial side, a high dose is prescribed to the tumor region and upper limits are put on the OAR dose. A radiation intensity profile (fluence-map) that conforms to this protocol as closely as possible is then found by solving an optimization problem. Intensity Modulated Radiation Therapy (IMRT) and well-established optimization models and efficient solution algorithms can now be employed to attain highly conformal dose profiles [7, 21].

Radiation is typically delivered over multiple sessions. One motivation for such fractionation is that healthy cells often have better damage-repair capabilities than tumor cells. Thus, breaking the prescribed dose into well-separated sessions allows the healthy cells to recover between sessions. A large number of fractions with a small dose per fraction thus may allow more dose and hence cause more damage to the tumor as compared to administering a small number of fractions with a large dose per fraction. On the flip side, tumors can proliferate over the treatment course, and thus a shorter course might be better as it kills the tumor before any significant proliferation. These trade-offs are often referred to as the fractionation problem, which involves finding the optimal number of sessions and the corresponding doses in these sessions.

Most mathematical formulations of the fractionation problem rely on the well-known linearquadratic (LQ) model of dose-response for tumor and OAR. The prevalent theme in the existing literature on these formulations is to separate the spatial and temporal components. That is, a fluence-map is assumed to be known a priori from a spatial treatment planning system for IMRT.

\footnotetext{
${ }^{*}$ Corresponding author: Deaprtment of Industrial and Systems Engineering, Box 352650, University of Washington, Seattle, WA, 98195, USA; archis@uw.edu.
} 
The number of treatment sessions and corresponding doses are then optimized (by appropriately scaling the pre-determined fluence-map) using a biological objective function and biological constraints that explicitly incorporate the dose-response behavior of tumor and OAR via the LQ model. We refer the reader, for example, to $[2,5,6,8,9,10,12,13,15,17,19,20$, 22] for discussions, analyses, and solutions of such spatiotemporally separated formulations.

The theme in these separated formulations is to maximize the biological effect (BE) of radiation dose on tumor subject to upper bounds on the biologically effective dose (BED) to OAR. This leads to a nonconvex quadratically constrained quadratic programming (QCQP) model. Most of these QCQP formulations incorporate only a single OAR and then provide a closed-form solution for the optimal number of sessions and the corresponding tumor-dose per session. Two recent formulations considered two OAR; the first one [22] employed the simulated annealing heuristic for optimization, and the second [5] provided Karush-Kuhn-Tucker conditions for optimality. Saberian et al. [17] were the first to even partly analyze the case of multiple OAR. They derived closed-form solutions when the so-called $\alpha$ and $\beta$ parameters of the LQ dose-response models for the tumor and OAR were ordered in a certain way. Saberian et al. [19] later established the surprising result that the nonconvex QCQP can be equivalently reformulated as a two-variable linear program (LP). This provided the first provably optimal complete solution of the fractionation problem.

Most recently, Saberian et al. [18] proposed a spatiotemporally integrated formulation of the fractionation problem, where the number of sessions and the fluence-map were both optimization variables within a single model. The objective was again to maximize the tumor-BE subject to upper bounds on the BED for various OAR. Unlike the separated case, this problem cannot be equivalently reformulated as a two-variable LP. They therefore devised an approximate solution algorithm based on convex programming and demonstrated, via extensive numerical experiments, that such integrated optimization improves the tumor-BE compared to the separated model.

A key limitation of the above research on the fractionation problem is that it ignores the uncertainty in the values of the $\alpha$ and $\beta$ parameters of the LQ dose-response model. Recent studies about the spatiotemporally separated formulations of the fractionation problem have addressed this concern. Both Badri et al. [3] and Ajdari and Ghate [1] (unpublished), independently of each other, investigated a robust counterpart of the separated formulation from Saberian et al. [19]. In the robust optimization parlance, they assumed that the so-called $\alpha / \beta$ ratios of parameters of the LQ dose-response model for OAR belong to an interval uncertainty set. For instance, Ajdari and Ghate showed that an optimal solution to the nonconvex robust problem can be recovered by instead solving a small group of two-variable LPs. Each of these LPs is similar to the two-variable LP originally proposed by Saberian et al. [19] for solving the nonconvex QCQP formulation of the nominal problem. Badri et al. also developed a chance constrained approach to accommodate uncertainty in the $\alpha / \beta$ ratios in the separated model.

We introduce a robust counterpart of the spatiotemporally integrated fractionation problem. We assume that the unknown parameters of the LQ dose-response model belong to known intervals. The goal in the robust problem is then to ensure that its solution remains feasible for all values of the dose-response parameters from these intervals. We demonstrate that the resulting robust optimization problem can be equivalently reformulated as a group of problems with linear objectives and convex quadratic constraints. It is thus efficiently solvable. We remark as an aside that this reformulation method is more general than and hence applicable to our aforementioned unpublished work on the robust spatiotemporally separated formulation [1]. We numerically quantify the price of robustness for a representative test case for head-and-neck cancer from [18], and also compare the frequency and amount of infeasibility suffered by the nominal and the robust solutions. Our results suggest that the price of robustness is small and the robust solution remains statistically more feasible than the nominal solution even when dose-response parameters vary outside the presumed 
intervals of uncertainty. Since the uncertainty in dose-response parameters has been one of the hurdles in widespread use of nominal optimization formulations based on the LQ model, these numerical observations bode well for the potential clinical utility of their robust counterparts.

\section{Nominal and robust formulations}

We first briefly recall the nominal spatiotemporally integrated formulation from [18]. The objective in this formulation is to choose the number of sessions and the fluence-map in each session to maximize tumor-BE subject to maximum dose and mean dose constraints on various OAR. Dosevolume constraints can also be handled using the approach described in [18]; we omit those here for brevity. The formulation also includes smoothness constraints on the fluence-map to ensure that it is deliverable in practice using IMRT. Here, we only discuss minimal, crucial ingredients of this formulation; the reader is referred to [18] for an extensive discussion of its derivation, advantages, and limitations.

\subsection{Review of the nominal formulation from [18]}

Let $n$ denote the number of tumor voxels. The radiation field is discretized into small segments called beamlets. Let $k$ denote the number of beamlets. Let $u \in \Re_{+}^{k}$ denote the $k$-dimensional fluence-map employed in each session. The number of treatment sessions is denoted by $N$, with $N_{\max }$ being the maximum number of sessions allowed in the protocol. Let $A$ denote the $n \times k$ nonnegative tumor dose-deposition matrix; $A_{i}$ denotes its $i$ th row, which corresponds to the $i$ th tumor voxel. According to the linear dose-deposition model, $A_{i} u$ is the dose delivered to the $i$ th tumor voxel in each session. Moreover, let $\bar{A} u \triangleq \sum_{i=1}^{n} A_{i} u / n$ be the average dose over all tumor voxels in each session. Let $S$ denote the matrix employed in writing smoothness constraints on $u$.

Let $\alpha_{0}$ and $\beta_{0}$ denote the tumor-response parameters. A tumor proliferation term is also included in our model; it is defined by $\tau(N) \triangleq\left(\left[(N-1)-T_{\text {lag }}\right]^{+} \ln 2\right) / T_{\text {double, }}$, where $\left[(N-1)-T_{\text {lag }}\right]^{+}=$ $\max \left((N-1)-T_{\text {lag }}, 0\right)$. In this formula, $T_{\text {lag }}$ represents the time (in days) after which the tumor starts proliferating following the start of treatment and $T_{\text {double }}$ is the tumor doubling time (in days).

The set of OAR is denoted by $\mathcal{M} \triangleq \mathcal{M}_{1} \cup \mathcal{M}_{2}$; here, $\mathcal{M}_{1}$ and $\mathcal{M}_{2}$ are mutually exclusive sets of OAR with maximum dose and mean dose constraints, respectively. We use the index $m$ to denote quantities related to OAR $m \in \mathcal{M}$. The set of voxels in OAR $m$ is denoted by $\mathcal{N}_{m} \triangleq\left\{1,2, \ldots, n_{m}\right\}$. Let $\rho_{m} \triangleq \beta_{m} / \alpha_{m}$ denote the inverse alpha-over-beta ratio of the $\alpha$ and $\beta$ parameters of the LQ dose-response model for OAR $m$. Suppose for OAR $m \in \mathcal{M}_{1}$ that a total dose $D_{\max }^{m}$ is known to be tolerated by each voxel if administered in $N_{\text {conv }}^{m}$ equal-dose fractions. Similarly, suppose for OAR $m \in \mathcal{M}_{2}$ that total mean dose $D_{\text {mean }}^{m}$ is known to be tolerated if administered in $N_{\text {conv }}^{m}$ equal-dose fractions. Let $\mathrm{BED}_{\square}^{m}=D_{\square}^{m}+\rho_{m}\left(D_{\square}^{m}\right)^{2} / N_{\text {conv }}^{m}$ be the BED of total dose $D_{\square}^{m}$ if administered in $N_{\text {conv }}^{m}$ equal-dose fractions, where $\square$ represents either max or mean.

The nominal spatiotemporally integrated formulation is written in [18] as

$$
\begin{aligned}
(P) F^{*}=\max _{N, u} N \alpha_{0}(\bar{A} u) & +N \beta_{0}(\bar{A} u)^{2}-\tau(N), \\
N\left(A_{j}^{m} u\right)+N \rho_{m}\left(A_{j}^{m} u\right)^{2} & \leq \mathrm{BED}_{\text {max }}^{m}, \forall j \in \mathcal{N}_{m}, m \in \mathcal{M}_{1}, \\
N \sum_{j=1}^{n_{m}}\left(A_{j}^{m} u\right)+N \rho_{m} \sum_{j=1}^{n_{m}}\left(A_{j}^{m} u\right)^{2} & \leq n_{m} \mathrm{BED}_{\text {mean }}^{m}, m \in \mathcal{M}_{2}, \\
S u & \leq 0,
\end{aligned}
$$




$$
u \geq 0,1 \leq N \leq N_{\max }, \text { integer. }
$$

The objective in (1) is to maximize the $\mathrm{BE}$ of average tumor dose. Constraints (2) enforce that the BED to each OAR voxel is no more than the conventional BED; these are called the maximum dose constraints. Constraints (3) imply that the average BED of doses administered to different voxels is bounded above by the conventional BED; these are the mean dose constraints. Constraint (4) ensures, via an appropriately constructed smoothness matrix $S$, that the relative absolute difference between geometrically adjacent components of $u$ is within a range that IMRT can attain.

\subsection{Robust counterpart of the nominal model}

Let $\tilde{\rho}_{m}$ represent the "true" unknown value of $\rho_{m}$ for each OAR $m \in \mathcal{M}$. We assume that $\tilde{\rho}_{m}$ belongs to the uncertainty interval $\left[\rho_{m}^{\min }, \rho_{m}^{\max }\right]$, where $0 \leq \rho_{m}^{\min } \leq \rho_{m}^{\max }$. Thus, by replacing $\rho_{m}$ with $\tilde{\rho}_{m}$ and expanding $\mathrm{BED}_{\square}^{m}$ in the nominal formulation, the robust counterpart of $(P)$ is

$$
\begin{gathered}
(R P) \max _{N, u} N \alpha_{0}(\bar{A} u)+N \beta_{0}(\bar{A} u)^{2}-\tau(N), \\
N\left(A_{j}^{m} u\right)+\tilde{\rho}_{m}\left(N\left(A_{j}^{m} u\right)^{2}-\frac{\left(D_{\text {max }}^{m}\right)^{2}}{N_{\text {conv }}^{m}}\right) \leq D_{\max }^{m}, \forall j \in \mathcal{N}_{m}, m \in \mathcal{M}_{1}, \rho_{m}^{\min } \leq \tilde{\rho}_{m} \leq \rho_{m}^{\max }, \\
N \sum_{j=1}^{n_{m}}\left(A_{j}^{m} u\right)+\tilde{\rho}_{m}\left(N \sum_{j=1}^{n_{m}}\left(A_{j}^{m} u\right)^{2}-\frac{n_{m}\left(D_{\text {mean }}^{m}\right)^{2}}{N_{\text {conv }}^{m}}\right) \leq n_{m} D_{\text {mean }}^{m}, m \in \mathcal{M}_{2}, \rho_{m}^{\min } \leq \tilde{\rho}_{m} \leq \rho_{m}^{\max },
\end{gathered}
$$

$$
(4)-(5) \text {. }
$$

Here, inequalities (7)-(8) ensure that the maximum dose and the mean dose constraints are respected for every possible realization of $\tilde{\rho}_{m}$ in the interval $\left[\rho_{m}^{\min }, \rho_{m}^{\max }\right]$. Consequently, they include an uncountably infinite number of constraints, and thus this robust problem appears intractable at first glance. Fortunately, it can be equivalently rewritten in a simple form by applying the general method proposed in Section 2.2 of [4] for robust optimization under polyhedral uncertainty. Below we convey the main idea in this reformulation using the mean dose constraints (8).

For any fixed $u$ and $N$, the mean dose constraint holds for every realization of $\tilde{\rho}_{m}$ if and only if the maximum of the left hand side in inequality (8) over all $\tilde{\rho}_{m} \in\left[\rho_{m}^{\min }, \rho_{m}^{\max }\right]$ is no more than the right hand side $n_{m} D_{\text {mean }}^{m}$. For any fixed $u$ and $N$, the problem of maximizing this left hand side is an LP in variables $\tilde{\rho}_{m}$ with only two constraints: $\tilde{\rho}_{m} \leq \rho_{m}^{\max }$ and $\tilde{\rho}_{m} \geq \rho_{m}^{\min }$. By attaching variables $q_{m}$ and $p_{m}$, respectively, with these two constraints, we can write the dual of this LP. This dual is a minimization problem, and by strong duality its optimal cost equals the maximum value in the primal LP. In other words, For any fixed $u$ and $N$, the mean dose constraint holds for every realization of $\tilde{\rho}_{m}$ if and only if the optimal cost in the dual LP is no more than $n_{m} D_{\text {mean }}^{m}$. This allows us to replace the robust mean dose constraints with another equivalent group of constraints. This idea can be expressed mathematically as follows:

$$
\begin{gathered}
\max _{\rho_{m}^{\min } \leq \tilde{\rho}_{m} \leq \rho_{m}^{\max }}\left\{N \sum_{j=1}^{n_{m}}\left(A_{j}^{m} u\right)+\tilde{\rho}_{m}\left(N \sum_{j=1}^{n_{m}}\left(A_{j}^{m} u\right)^{2}-n_{m} \frac{\left(D_{\text {mean }}^{m}\right)^{2}}{N_{\text {conv }}^{m}}\right)\right\} \leq n_{m} D_{\text {mean }}^{m} \\
\Leftrightarrow\left(\begin{array}{c}
\min _{q_{m}, p_{m}} N \sum_{j=1}^{n_{m}}\left(A_{j}^{m} u\right)+\rho_{m}^{\max } q_{m}+\rho_{m}^{\min } p_{m}, \\
q_{m}+p_{m} \geq N \sum_{j=1}^{n_{m}}\left(A_{j}^{m} u\right)^{2}-n_{m} \frac{\left(D_{\text {mean }}^{m}\right)^{2}}{N_{\text {conv }}^{m}}, \\
q_{m} \geq 0, p_{m} \leq 0 .
\end{array}\right) \leq n_{m} D_{\text {mean }}^{m} .
\end{gathered}
$$


Applying this to maximum dose constraints as well, the robust optimization problem becomes

$$
\begin{gathered}
(R P) F^{*}=\max _{N, u, \mathrm{q}, \mathrm{p}} N \alpha_{0}(\bar{A} u)+N \beta_{0}(\bar{A} u)^{2}-\tau(N), \\
\text { s.t. } N\left(A_{j}^{m} u\right)+\rho_{m}^{\max } q_{m}^{j}+\rho_{m}^{\min } p_{m}^{j} \leq D_{\max }^{m}, \forall j \in \mathcal{N}_{m}, m \in \mathcal{M}_{1}, \\
N\left(A_{j}^{m} u\right)^{2}-q_{m}^{j}-p_{m}^{j} \leq \frac{\left(D_{\text {max }}^{m}\right)^{2}}{N_{\text {conv }}^{m}}, \forall j \in \mathcal{N}_{m}, m \in \mathcal{M}_{1}, \\
N \sum_{j=1}^{n_{m}}\left(A_{j}^{m} u\right)+\rho_{m}^{\max } q_{m}+\rho_{m}^{\min } p_{m} \leq n_{m} D_{\text {mean }}^{m}, \forall m \in \mathcal{M}_{2}, \\
N \sum_{j=1}^{n_{m}}\left(A_{j}^{m} u\right)^{2}-q_{m}-p_{m} \leq n_{m} \frac{\left(D_{\text {mean }}^{m}\right)^{2}}{N_{\text {conv }}^{m}}, \forall m \in \mathcal{M}_{2}, \\
p \leq 0, q \geq 0,(4)-(5) .
\end{gathered}
$$

Here, $q$ and $p$ are the vectors of all dual variables.

For each fixed $N,(R P)$ maximizes a convex function of $u$. This difficulty can be addressed by further simplifying $(R P)$ as in the nominal formulation from [18]. First, we propose to solve the robust problem for each fixed integer value of $N$ in the range $1 \leq N \leq N_{\text {max }}$. We call these problems $(R P(N))$ and denote their optimal values by $F^{*}(N)$. The optimal value and solution for $(R P)$ can then be found simply by choosing the best among all $(R P(N))$. Furthermore, for each fixed $N$, the objective in $(R P(N))$ is increasing in $\bar{A} u$. Thus, maximizing this objective is equivalent to maximizing $\bar{A} u$. We utilize this in solving $(R P(N))$. Consequently, we note that each $(R P(N))$ includes an objective that is linear in $u$ and that does not include variables $p, q$. Finally, constraints in each $(R P(N))$ are convex quadratic in $u$ and linear in $p, q$. As such, problems $(R P(N))$ can be solved efficiently in practice using off-the-shelf software.

\section{Results}

\subsection{Description of a head-and-neck cancer case}

We demonstrate our numerical results on a representative head-and-neck cancer case from [18]. The case included four OAR: spinal cord, brainstem, left and right parotids. The nominal values of $\rho$ for all OAR were fixed at $\rho=1 / 3 \mathrm{~Gy}^{-1}$ based on $[8,22]$. We followed a treatment protocol similar to QUANTEC [14]. Specifically, the conventional number of sessions $N_{\text {conv }}$ was fixed at 35. Maximum dose constraints of 45 Gy and 50 Gy were included for spinal cord and brainstem, respectively. Mean dose constraints with a tolerance dose of 28 Gy were included for left and right parotids. $N_{\max }$ was set to 100 days. We are aware that this value is somewhat unrealistically high; we nevertheless used it to bring forth the full range of sensitivity behaviors in our problem. Tumor-response parameters were fixed at $\alpha_{0}=0.35 \mathrm{~Gy}^{-1}$ and $\beta_{0}=0.035 \mathrm{~Gy}^{-2}$ based on $[8,9]$. A maximum dose of 90 Gy was enforced on all tumor voxels to encourage dose-uniformity. The fluence-map $u$ consisted of 3,910 beamlets and the total number of constraints in the nominal formulation was 27,450. All computer simulations were performed on a $3.1 \mathrm{GHz}$ iMac desktop with 16 GB RAM using the convex optimization toolbox CVX [11] in MATLAB.

\subsection{Price of robustness}

Price of robustness is defined as the relative decrease in the optimal tumor BE in the robust formulation compared to the nominal formulation. In our numerical experiments, uncertainty 


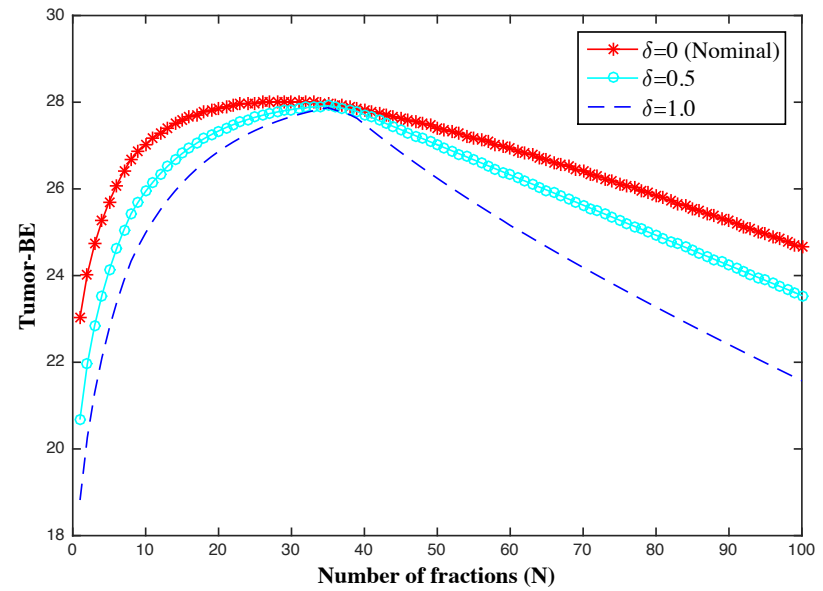

(a)

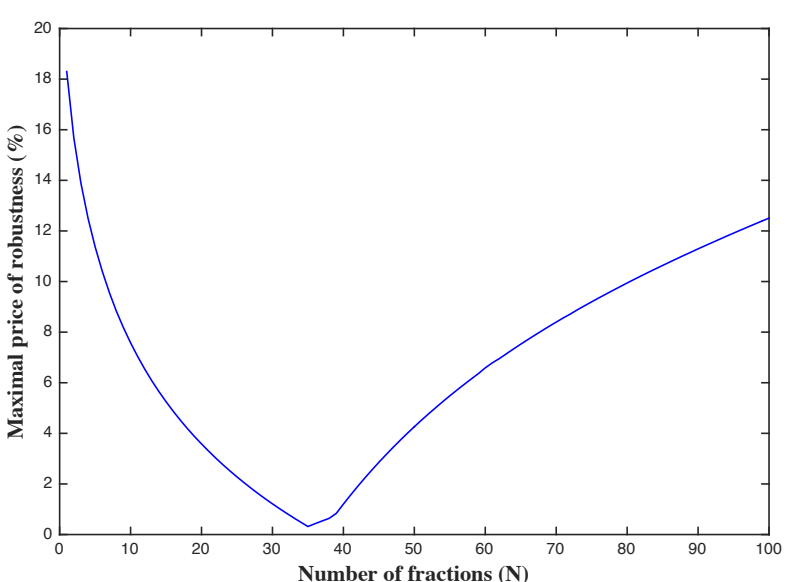

(b)

Figure 1: (a) Tumor-BE as a function of $N$. (b) Maximal price of robustness (\%) as a function of $N$. This was calculated as $100 \times\left(\mathrm{BE}_{\text {nominal }}-\mathrm{BE}_{\delta=1}\right) / \mathrm{BE}_{\text {nominal }}$.

intervals were parameterized as $[(1-\delta) / 3,(1+\delta) / 3]$, with $\delta \in\{0,0.1, \ldots, 1\}$, where $(1 / 3) \mathrm{Gy}^{-1}$ is the nominal value of $\rho$ for all OAR. Here, $\delta=0$ corresponds to the nominal case, $\delta=1$ represents $100 \%$ uncertainty. This allowed us to quantify the price of robustness as a function of the single uncertainty parameter $\delta$.

Tables 1 and 2 summarize the results of 200 experiments for different values of $T_{\text {lag }}, T_{\text {double }}$, and $\delta$. In these experiments, $T_{\text {lag }}$ values were set to $7,14,21,28$ days based on [9] and $T_{\text {double values }}$ were set to $2,10,20,40,50$ days based on $[8,9,16,22]$.

Table 1 shows, as expected, that the price of robustness increases with increasing $\delta$ for each $T_{\text {lag }}, T_{\text {double }}$ combination. Overall, the price of robustness was small with an average of $1.29 \%$, with first, second, and third quartiles of $0.48 \%, 0.98 \%$, and $1.66 \%$, respectively.

For all $T_{\text {lag }}, \delta$ combinations in Table 1 , the price of robustness first decreases with increasing $T_{\text {double }}$, reaches its smallest value, and then increases. This trend is consistent with the corresponding trend in the difference between $N_{\text {conv }}^{m}=35$ and the optimal number of fractions $\left(N^{*}\right)$ that can be inferred from Table 2. Specifically, the absolute difference $\left|N_{\mathrm{conv}}^{m}-N^{*}\right|$ decreases with increasing $T_{\text {double }}$, reaches its minimum, and then increases.

Figure 1a shows the evolution of tumor-BE with $N$ for $T_{\text {lag }}=7$ days and $T_{\text {double }}=10$ days as an example. The price of robustness equals zero at 35 because when $N=N_{\text {conv }}^{m}, \rho_{m}$ is eliminated from the BED constraints thus reducing them to total dose constraints. In other words, the nominal and the robust problems become identical. Figure $1 \mathrm{~b}$ shows the trend in the maximal price of robustness as an example.

As can be seen from Table 1, there is no universal trend for how the price of robustness changes as $T_{\text {lag }}$ increases. For example, when $T_{\text {double }}=2$ and $\delta=0.1$, the price of robustness decreases as $T_{\text {lag }}$ increases; whereas when $T_{\text {double }}=2$ and $\delta=0.7$, the price of robustness first decreases from $T_{\text {lag }}=7$ to 14 , then increases when $T_{\text {lag }}=21$, and then decreases again. However, in all cases, the change in the price of robustness with $T_{\text {lag }}$ is consistent with the trend in $\left|N_{\text {conv }}^{m}-N^{*}\right|$, as can be inferred from Table 2 .

A closer look at Table 2 reveals that the evolution of $N^{*}$ with $\delta$ does not exhibit a universal trend. For instance, $N^{*}$ increases with increasing $\delta$ when $T_{\text {double }}=2$ and 10 days, and decreases as $\delta$ increases when $T_{\text {double }}=20,40$ and 50 days.

Our robust solutions continue to exhibit qualitative trends that are well-established in the 
nominal case. For instance, $N^{*}$ increases with increasing $T_{\text {double }}$ for any fixed $\delta, T_{\text {lag }}$ combination. Similarly, $N^{*}$ also increases as $T_{\text {lag }}$ increases for any fixed $\delta, T_{\text {double }}$ combination.

\subsection{Infeasibility tests}

The nominal solution is guaranteed to remain feasible only when the realized value of the $\rho$ parameters equal their nominal values. To quantify the frequency and the amount of infeasibility of the nominal solutions when this is not the case, we computed the fluence-map and the number of sessions that would be optimal if the dose-response parameters equaled their nominal values, and tested whether or not such a nominal solution would remain feasible if the true dose-parameters took some other values from the uncertainty interval. For each uncertainty level $\delta$ and for each constraint $m$, five different grid-points were selected to calculate the realized values of dose-parameters. The five values were $\tilde{\rho}_{m}=\rho_{m}^{\min }+2 i \rho_{m} \delta / 5$, where $i=1,2, \ldots, 5$, and $\rho_{m}$ denotes the nominal value $\left(1 / 3 \mathrm{~Gy}^{-1}\right)$ of the dose-parameter for constraint $m$. We performed this experiment for all combinations of $T_{\text {lag }}, T_{\text {double, }}$, and $\delta$. We call the nominal solution infeasible if it violates at least one constraint. In this sense, the nominal solution was infeasible in over $91 \%$ of the cases. The amount of maximum infeasibility itself was as large as $70 \%$ in some cases, with an average of $10.11 \%$ and first, second, third quartiles of $3.20 \%, 7.12 \%$, and $15.56 \%$. In all cases, the robust solutions of course remained feasible.

The fundamental assumption in developing our robust formulation was that the true values of the dose-parameters, although uncertain, belonged to some presumed uncertainty set. This assumption however might not hold. Therefore, to quantify the frequency and amount of infeasibility for robust and nominal solutions when dose-response parameters varied outside the uncertainty intervals, we repeated the above experiments by changing parameter values as follows: for each uncertainty level $\delta$ and constraint $m$, five grid-points were chosen at $\tilde{\rho}_{m}=(1+\delta+\gamma) \rho_{m}$ and five grid-points at $\tilde{\rho}_{m}=(1-\delta-\gamma) \rho_{m}$, where $\gamma \in\{0.1,0.2, \ldots, 0.5\}$ and $\rho_{m}$ denotes the nominal value of $1 / 3 \mathrm{~Gy}^{-1}$. We performed numerical experiments for all combinations of $T_{\text {lag }}, T_{\text {double }}$, and $\delta$. The nominal solution was infeasible in over $76 \%$ of the cases, while the robust solution was infeasible in $58 \%$ of the cases. Furthermore, the average amount of maximum infeasibility was less for the robust solution than for the nominal (6.84\% versus $12.19 \%$ ). A pairwise t-test revealed that this difference was significant at the $95 \%$ level. This suggests that the robust solution is statistically less infeasible than the nominal.

\subsection{Uncertainty in tumor parameters $\alpha_{0}$ and $\beta_{0}$}

We assumed for simplicity that there is no uncertainty in the tumor-response parameters $\alpha_{0}$ and $\beta_{0}$. It is easy to relax this assumption while writing the robust counterpart of $(P)$. This is because, under interval uncertainty for parameters $\alpha_{0}$ and $\beta_{0}$, the worst-case objective value is obtained simply by inserting the worst values of these parameters. Specifically, we now assume that true values $\tilde{\alpha}_{0}$ and $\tilde{\beta}_{0}$ of these parameters belong to known intervals $\left[\alpha_{0}^{\min }, \alpha_{0}^{\max }\right]$ and $\left[\beta_{0}^{\min }, \beta_{0}^{\max }\right]$, respectively. Then, the objective function of the robust problem is given by replacing $\tilde{\alpha}_{0}$ with $\alpha_{0}^{\min }$ and $\tilde{\beta}_{0}$ with $\beta_{0}^{\min }$. Recall that the treatment protocol described in Section 3.1 also included maximum dose constraints on tumor voxels. The robust counterpart of these constraints under interval uncertainty was derived in our numerical experiments below by applying the procedure described in Section 2.2 for maximum dose constraints on OAR.

We characterized the interval uncertainty in tumor-response parameters via a single parameter by setting $\alpha_{0}^{\min }=(1-\theta) 0.35 \mathrm{~Gy}^{-1} ; \alpha_{0}^{\max }=(1+\theta) 0.35 \mathrm{~Gy}^{-1} ; \beta_{0}^{\min }=(1-\theta) 0.035 \mathrm{~Gy}^{-2}$; and $\beta_{0}^{\max }=(1+\theta) 0.035 \mathrm{~Gy}^{-2}$, for $\theta \in\{0.1,0.2, \ldots, 0.9\}$. Table 3 shows the results for $T_{\text {lag }}=7$ days 
and $T_{\text {double }}=21$ days as a representative example. For each fixed $\theta$, the qualitative trend in the evolution of $N^{*}$ was similar to the previous section. By dividing the objective function in the robust problem by $(1-\theta)$, we see that $(1-\theta)$ scales $T_{\text {double }}$ in the denominator of the proliferation term $\tau(N)$. Consequently, a larger $\theta$ has the same effect on BE as a faster growing tumor (that is, a smaller $\left.T_{\text {double }}\right)$. Thus, a larger $\theta$ should lead to a shorter treatment course. This trend can be observed in each row of Table 3. Finally, the price of robustness (not listed in the table for brevity) was insensitive to $\theta$, and remained small with an average of $0.87 \%$ over all $\delta, \theta$ combinations.

\section{Conclusion}

There is a large body of literature on robust spatial optimization in radiotherapy, where the goal is to compensate for intra-fraction and inter-fraction errors made in dose-delivery due to patient movement and patient setup. Here, we applied robust optimization to a different problem that of incorporating uncertainty in dose-response parameters in deciding fractionation schedules. Although the robust counterpart of this fractionation problem first appeared intractable, we were able to equivalently reformulate it as a simple convex problem that can be solved efficiently. Our numerical experiments on a representative head-and-neck test case suggest that the resulting price of robustness is small and that the robust solutions are statistically less infeasible than nominal. This bodes well for potential clinical implementation of our robust optimization methodology.

\section{Acknowledgment}

Funded in part by the National Science Foundation through grant \#CMMI 1054026.

\begin{tabular}{|c|c|c|c|c|c|c|c|c|c|c|c|}
\hline$T_{\mathrm{lag}}=7$ & \multicolumn{5}{|c|}{$T_{\text {double }}$} & $T_{\mathrm{lag}}=14$ & \multicolumn{5}{|c|}{$T_{\text {double }}$} \\
\hline & 2 & 10 & 20 & 40 & 50 & $\delta$ & 2 & 10 & 20 & 40 & 50 \\
\hline 0.1 & 1.47 & 0.17 & 0.1 & 0.51 & 0.61 & 0.1 & 0.61 & 0.27 & 0.1 & 0.5 & 0.61 \\
\hline 0.2 & 2.8 & 0.26 & 0.16 & 0.92 & 1.15 & 0.2 & 1.15 & 0.43 & 0.16 & 0.92 & 1.14 \\
\hline 0.3 & 4.01 & 0.3 & 0.21 & 1.17 & 1.54 & 0.3 & 1.54 & 0.52 & 0.21 & 1.16 & 1.54 \\
\hline 0.4 & 5.11 & 0.33 & 0.27 & 1.24 & 1.66 & 0.4 & 1.66 & 0.56 & 0.26 & 1.23 & 1.66 \\
\hline 0.5 & 6.12 & 0.37 & 0.32 & 1.31 & 1.73 & 0.5 & 1.73 & 0.6 & 0.32 & 1.3 & 1.73 \\
\hline 0.6 & 7.01 & 0.4 & 0.39 & 1.39 & 1.81 & 0.6 & 1.81 & 0.63 & 0.39 & 1.38 & 1.81 \\
\hline 0.7 & 7.81 & 0.43 & 0.45 & 1.46 & 1.9 & 0.7 & 1.9 & 0.66 & 0.45 & 1.46 & 1.89 \\
\hline 0.8 & 8.54 & 0.46 & 0.48 & 1.54 & 1.97 & 0.8 & 1.97 & 0.69 & 0.48 & 1.53 & 1.97 \\
\hline 0.9 & 9.19 & 0.49 & 0.51 & 1.63 & 2.06 & 0.9 & 2.06 & 0.72 & 0.51 & 1.62 & 2.06 \\
\hline 1 & 9.8 & 0.51 & 0.54 & 1.71 & 2.17 & 1 & 2.17 & 0.75 & 0.53 & 1.71 & 2.16 \\
\hline$T_{\text {lag }}=21$ & \multicolumn{5}{|c|}{$\overline{T_{\text {double }}}$} & $T_{\text {lag }}=28$ & \multicolumn{5}{|c|}{$T_{\text {double }}$} \\
\hline$\delta$ & 2 & 10 & 20 & 40 & 50 & $\delta$ & 2 & 10 & 20 & 40 & 50 \\
\hline 0.1 & 0.47 & 0.27 & 0.1 & 0.5 & 0.61 & 0.1 & 0.21 & 0.21 & 0.1 & 0.5 & 0.6 \\
\hline 0.2 & 0.89 & 0.43 & 0.16 & 0.92 & 1.14 & 0.2 & 0.41 & 0.37 & 0.16 & 0.91 & 1.14 \\
\hline 0.3 & 1.29 & 0.51 & 0.21 & 1.16 & 1.53 & 0.3 & 0.59 & 0.45 & 0.21 & 1.15 & 1.53 \\
\hline 0.4 & 1.65 & 0.55 & 0.26 & 1.23 & 1.65 & 0.4 & 0.75 & 0.49 & 0.26 & 1.22 & 1.65 \\
\hline 0.5 & 1.99 & 0.59 & 0.32 & 1.3 & 1.72 & 0.5 & 0.91 & 0.53 & 0.32 & 1.29 & 1.72 \\
\hline 0.6 & 2.31 & 0.62 & 0.38 & 1.38 & 1.8 & 0.6 & 1.05 & 0.56 & 0.38 & 1.37 & 1.8 \\
\hline 0.7 & 2.6 & 0.65 & 0.44 & 1.45 & 1.89 & 0.7 & 1.19 & 0.59 & 0.44 & 1.44 & 1.88 \\
\hline 0.8 & 2.88 & 0.68 & 0.48 & 1.53 & 1.96 & 0.8 & 1.32 & 0.62 & 0.47 & 1.52 & 1.96 \\
\hline 0.9 & 3.13 & 0.71 & 0.5 & 1.62 & 2.05 & 0.9 & 1.44 & 0.65 & 0.5 & 1.61 & 2.04 \\
\hline 1 & 3.38 & 0.74 & 0.53 & 1.7 & 2.15 & 1 & 1.55 & 0.67 & 0.52 & 1.69 & 2.14 \\
\hline
\end{tabular}

Table 1: Price of robustness (\%) for different $T_{\text {lag }}$ (days), $T_{\text {double }}$ (days), and uncertainty levels $\delta$. 


\begin{tabular}{|c|ccccc|}
\hline$T_{\text {lag }}=7$ & \multicolumn{5}{|c|}{$T_{\text {double }}$} \\
$\delta$ & 2 & 10 & 20 & 40 & 50 \\
\hline 0 & $(6.03,8)$ & $(2.46,28)$ & $(1.68,44)$ & $(1.12,71)$ & $(0.98,82)$ \\
0.1 & $(5.97,8)$ & $(2.35,29)$ & $(1.85,39)$ & $(1.25,62)$ & $(1.09,73)$ \\
0.2 & $(5.91,8)$ & $(2.17,32)$ & $(1.85,39)$ & $(1.43,53)$ & $(1.26,61)$ \\
0.3 & $(5.86,8)$ & $(2.07,34)$ & $(1.89,38)$ & $(1.78,41)$ & $(1.58,47)$ \\
0.4 & $(5.81,8)$ & $(2.02,35)$ & $(1.89,38)$ & $(1.85,39)$ & $(1.85,39)$ \\
0.5 & $(5.76,8)$ & $(2.02,35)$ & $(1.89,38)$ & $(1.85,39)$ & $(1.85,39)$ \\
0.6 & $(5.30,9)$ & $(2.02,35)$ & $(1.89,38)$ & $(1.85,39)$ & $(1.85,39)$ \\
0.7 & $(5.27,9)$ & $(2.02,35)$ & $(1.98,36)$ & $(1.89,38)$ & $(1.89,38)$ \\
0.8 & $(4.89,10)$ & $(2.02,35)$ & $(2.02,35)$ & $(1.89,38)$ & $(1.89,38)$ \\
0.9 & $(4.86,10)$ & $(2.02,35)$ & $(2.02,35)$ & $(1.89,38)$ & $(1.89,38)$ \\
1 & $(4.84,10)$ & $(2.02,35)$ & $(2.02,35)$ & $(2.02,35)$ & $(1.88,38)$ \\
\hline \hline$T_{\text {lag }}=14$ & & & $T_{\text {double }}$ & & \\
$\delta$ & 2 & 10 & 20 & 40 & 50 \\
\hline 0 & $(4.29,14)$ & $(2.57,26)$ & $(1.68,44)$ & $(1.12,71)$ & $(0.98,82)$ \\
0.1 & $(4.29,14)$ & $(2.35,29)$ & $(1.85,39)$ & $(1.25,62)$ & $(1.09,73)$ \\
0.2 & $(4.29,14)$ & $(2.17,32)$ & $(1.85,39)$ & $(1.43,53)$ & $(1.26,61)$ \\
0.3 & $(4.29,14)$ & $(2.07,34)$ & $(1.89,38)$ & $(1.78,41)$ & $(1.58,47)$ \\
0.4 & $(4.29,14)$ & $(2.02,35)$ & $(1.89,38)$ & $(1.85,39)$ & $(1.85,39)$ \\
0.5 & $(4.29,14)$ & $(2.02,35)$ & $(1.89,38)$ & $(1.85,39)$ & $(1.85,39)$ \\
0.6 & $(3.88,15)$ & $(2.02,35)$ & $(1.89,38)$ & $(1.85,39)$ & $(1.85,39)$ \\
0.7 & $(3.88,15)$ & $(2.02,35)$ & $(1.98,36)$ & $(1.89,38)$ & $(1.89,38)$ \\
0.8 & $(3.88,15)$ & $(2.02,35)$ & $(2.02,35)$ & $(1.89,38)$ & $(1.89,38)$ \\
0.9 & $(3.88,15)$ & $(2.02,35)$ & $(2.02,35)$ & $(1.89,38)$ & $(1.89,38)$ \\
1 & $(3.88,15)$ & $(2.02,35)$ & $(2.02,35)$ & $(2.02,35)$ & $(1.88,38)$ \\
\hline \hline$T_{\text {lag }}=21$ & & & $T_{\text {double }}$ & & \\
$\delta$ & 2 & 10 & 20 & 40 & 50 \\
\hline 0 & $(2.92,22)$ & $(2.57,26)$ & $(1.68,44)$ & $(1.12,71)$ & $(0.98,82)$ \\
0.1 & $(2.91,22)$ & $(2.35,29)$ & $(1.85,39)$ & $(1.25,62)$ & $(1.09,73)$ \\
0.2 & $(2.90,22)$ & $(2.17,32)$ & $(1.85,39)$ & $(1.43,53)$ & $(1.26,61)$ \\
0.3 & $(2.89,22)$ & $(2.07,34)$ & $(1.89,38)$ & $(1.78,41)$ & $(1.58,47)$ \\
0.4 & $(2.88,22)$ & $(2.02,35)$ & $(1.89,38)$ & $(1.85,39)$ & $(1.85,39)$ \\
0.5 & $(2.87,22)$ & $(2.02,35)$ & $(1.89,38)$ & $(1.85,39)$ & $(1.85,39)$ \\
0.6 & $(2.86,22)$ & $(2.02,35)$ & $(1.89,38)$ & $(1.85,39)$ & $(1.85,39)$ \\
0.7 & $(2.86,22)$ & $(2.02,35)$ & $(1.98,36)$ & $(1.89,38)$ & $(1.89,38)$ \\
0.8 & $(2.85,22)$ & $(2.02,35)$ & $(2.02,35)$ & $(1.89,38)$ & $(1.89,38)$ \\
0.9 & $(2.84,22)$ & $(2.02,35)$ & $(2.02,35)$ & $(1.89,38)$ & $(1.89,38)$ \\
1 & $(2.84,22)$ & $(2.02,35)$ & $(2.02,35)$ & $(2.02,35)$ & $(1.88,38)$ \\
\hline & & & & & \\
& & & & \\
& & & & \\
& & & & \\
0
\end{tabular}

Table 2: Optimal average dose per session (Gy) and the optimal number of sessions $\left(N^{*}\right)$ for $T_{\text {lag }}=7,14,21$ days.

\section{References}

[1] A Ajdari and A Ghate. Robust fractionation in radiotherapy. Unpublished, May 2015.

[2] C I Armpilia, R G Dale, and B Jones. Determination of the optimum dose per fraction in fractionated radiotherapy when there is delayed onset of tumour repopulation during treatment. The British Journal of Radiology, 77(921):765-767, 2004.

[3] H Badri, Y Watanabe, and K Leder. Robust and probabilistic optimization of dose schedules in radiotherapy. available online at arxiv.org/pdf/1503.00399v2.pdf, June 2015. 


\begin{tabular}{|c|ccccccccc|}
\hline & \multicolumn{8}{|c}{ Tumor } & \multicolumn{7}{c|}{ uncertainty level $(\theta)$} \\
$\delta$ & 0.1 & 0.2 & 0.3 & 0.4 & 0.5 & 0.6 & 0.7 & 0.8 & 0.9 \\
\hline 0 & $(1.78,41)$ & $(1.94,37)$ & $(2.07,34)$ & $(2.29,30)$ & $(2.57,26)$ & $(3.02,21)$ & $(3.54,17)$ & $(4.85,11)$ & $(6.03,8)$ \\
0.1 & $(1.85,39)$ & $(1.89,38)$ & $(2.03,35)$ & $(2.12,33)$ & $(2.35,29)$ & $(2.72,24)$ & $(3.25,19)$ & $(4.27,13)$ & $(5.97,8)$ \\
0.2 & $(1.89,38)$ & $(1.89,38)$ & $(2.02,35)$ & $(2.02,35)$ & $(2.17,32)$ & $(2.55,26)$ & $(3.00,21)$ & $(4.03,14)$ & $(5.91,8)$ \\
0.3 & $(1.89,38)$ & $(1.93,37)$ & $(2.02,35)$ & $(2.02,35)$ & $(2.07,34)$ & $(2.34,29)$ & $(2.89,22)$ & $(3.81,15)$ & $(5.86,8)$ \\
0.4 & $(1.89,38)$ & $(1.98,36)$ & $(2.02,35)$ & $(2.02,35)$ & $(2.02,35)$ & $(2.22,31)$ & $(2.70,24)$ & $(3.62,16)$ & $(5.81,8)$ \\
0.5 & $(1.89,38)$ & $(2.02,35)$ & $(2.02,35)$ & $(2.02,35)$ & $(2.02,35)$ & $(2.17,32)$ & $(2.61,25)$ & $(3.46,17)$ & $(5.76,8)$ \\
0.6 & $(1.98,36)$ & $(2.02,35)$ & $(2.02,35)$ & $(2.02,35)$ & $(2.02,35)$ & $(2.07,34)$ & $(2.46,27)$ & $(3.31,18)$ & $(5.30,9)$ \\
0.7 & $(2.02,35)$ & $(2.02,35)$ & $(2.02,35)$ & $(2.02,35)$ & $(2.02,35)$ & $(2.02,35)$ & $(2.39,28)$ & $(3.17,19)$ & $(5.27,9)$ \\
0.8 & $(2.02,35)$ & $(2.02,35)$ & $(2.02,35)$ & $(2.02,35)$ & $(2.02,35)$ & $(2.02,35)$ & $(2.33,29)$ & $(3.05,20)$ & $(4.89,10)$ \\
0.9 & $(2.02,35)$ & $(2.02,35)$ & $(2.02,35)$ & $(2.02,35)$ & $(2.02,35)$ & $(2.02,35)$ & $(2.27,30)$ & $(2.94,21)$ & $(4.86,10)$ \\
1 & $(2.02,35)$ & $(2.02,35)$ & $(2.02,35)$ & $(2.02,35)$ & $(2.02,35)$ & $(2.02,35)$ & $(2.21,31)$ & $(2.84,22)$ & $(4.84,10)$ \\
\hline
\end{tabular}

Table 3: Optimal average dose (Gy) per session and optimal number of sessions $\left(N^{*}\right)$ for various tumor uncertainty levels $(\theta)$ and OAR uncertainty levels $(\delta)$ for $T_{\text {lag }}=7$ days and $T_{\text {double }}=21$ days.

[4] D Bertsimas, D B Brown, and C Caramanis. Theory and applications of robust optimization. SIAM Review, 53(3):464-501, 2011.

[5] A Bertuzzi, C Bruni F Papa, and C Sinisgalli. Optimal solution for a cancer radiotherapy problem. Journal of Mathematical Biology, 66(1-2):311-349, 2013.

[6] T Bortfeld, J Ramakrishnan, J N Tsitsiklis, and J Unkelbach. Optimization of radiotherapy fractionation schedules in the presence of tumor repopluation. forthcoming in INFORMS Journal on Computing, prepring available at http://pages.discovery.wisc.edu/ jramakrishnan/BRT2015_repop.pdf, May 2015.

[7] M Ehrgott, Ç Güler, H W Hamacher, and L Shao. Mathematical optimization in intensity modulated radiation therapy. 4OR, 6(3):199-262, 2008.

[8] J F Fowler. How worthwhile are short schedules in radiotherapy? a series of exploratory calculations. Radiotherapy and Oncology, 18(2):165-181, 1990.

[9] J F Fowler. Optimum overall times II: Extended modelling for head and neck radiotherapy. Clinical Oncology, 20(2):113-126, 2008.

[10] J F Fowler and M A Ritter. A rationale for fractionation for slowly proliferating tumors such as prostatic adenocarcinoma. International Journal of Radiation Oncology Biology Physics, 32(2):521-529, 1995.

[11] M Grant and S Boyd. CVX: MATLAB software for disciplined convex programming (web page and software), 2009.

[12] B Jones, L T Tan, and R G Dale. Derivation of the optimum dose per fraction from the linear quadratic model. The British Journal of Radiology, 68(812):894-902, 1995.

[13] H Keller, G Meier, A Hope, and M Davison. Fractionation schedule optimization for lung cancer treatments using radiobiological and dose distribution characteristics. Medical Physics, 39(6):3811-3811, 2012.

[14] L B Marks, E D Yorke, A Jackson, R K Ten Haken, L S Constine, A Eisbruch, S M Bentzen, $\mathrm{J}$ Nam, and J O Deasy. Use of normal tissue complication probability models in the clinic. International Journal of Radiation Oncology Biology Physics, 76(3):S10-S19, 2010. 
[15] M Mizuta, S Takao, H Date, N Kishimoto, K L Sutherland, R Onimaru, and H Shirato. A mathematical study to select fractionation regimen based on physical dose distribution and the linear-quadratic model. International Journal of Radiation Oncology Biology Physics, 84(3):829-833, 2012.

[16] X S Qi, Q Yang, S P Lee, X A Li, and D Wang. An estimation of radiobiological parameters for head-and-neck cancer cells and the clinical implications. Cancers, 4:566-580, 2012.

[17] F Saberian, A Ghate, and M Kim. Optimal fractionation in radiotherapy with multiple normal tissues. forthcoming in Mathematical Medicine and Biology, online preprint available at doi: 10.1093/imammb/dqv015, May 2015.

[18] F Saberian, A Ghate, and M Kim. Spatiotemporally integrated fractionation in radiotherapy. under review at INFORMS Journal on Computing, preprint available at http://faculty . washington.edu/archis/upaper-apr-2015.pdf, April 2015.

[19] F Saberian, A Ghate, and M Kim. A two-variable linear program solves the standard linearquadratic formulation of the fractionation problem in cancer radiotherapy. Operations Research Letters, 43(3):254 - 258, 2015.

[20] J Unkelbach, D Craft, E Saleri, J Ramakrishnan, and T Bortfeld. The dependence of optimal fractionation schemes on the spatial dose distribution. Physics in Medicine and Biology, 58(1):159-167, 2013.

[21] S Webb. Contemporary IMRT: Developing Physics and Clinical Implementation. IOP Publishing, Bristol, UK, 2010.

[22] Y Yang and L Xing. Optimization of radiotherapy dose-time fractionation with consideration of tumor specific biology. Medical Physics, 32(12):3666-3677, 2005. 